\title{
Filter Diagonalization Method for Processing PFG NMR Data
}

\author{
Beau R. Martini and Vladimir A. Mandelshtam* \\ Chemistry Department, University of California at Irvine, Irvine, CA 92697, USA \\ Gareth A. Morris and Adam A. Colbourne \\ School of Chemistry, University of Manchester, Oxford Road, Manchester M13 9PL, United Kingdom
}

\author{
Mathias Nilsson \\ University of Copenhagen, \\ Faculty of Science, Dept. of Food Science, \\ Quality 85 Technology, Rolighedsvej 30, \\ DK-1958 Frederiksberg $C$, Denmark \\ and \\ School of Chemistry, University of Manchester, \\ Oxford Road, Manchester M13 9PL, United Kingdom
}

(Dated: June 17, 2013)

\begin{abstract}
Obtaining diffusion coefficients from PFG NMR diffusion (a.k.a DOSY) data is, in the general case, an ill-posed problem. Numerous methods for processing such data have therefore been developed, each with different constraints and assumptions. The Regularized Resolvent Transform (RRT) is a proven and robust method for spectral inversion. In earlier papers RRT, albeit very slow, was argued to be superior for DOSY processing to a related algorithm, the Filter Diagonalization Method (FDM). Here FDM is revisited and a new regularization model is implemented, which drastically improves the performance and provides spectra of comparable or better quality to those provided by RRT. Both the RRT and the FDM for DOSY processing have been implemented as options in the free and open source DOSY Toolbox.
\end{abstract}

\section{INTRODUCTION}

Arguably the most successful NMR method for mixture analysis is diffusion-ordered spectroscopy (DOSY). In DOSY, the different diffusion behavior of individual species is exploited to separate the NMR signals contributed by individual compounds [? ? ].

A DOSY data set is typically acquired using a pulsed field gradient spin or stimulated echo (PFGSE or PFGSTE) pulse sequence[? ? ] in which information on diffusion is obtained by recording spectra with different field gradient pulse amplitudes [? ? ? ? ? ]. A diffusion coefficient for each signal can normally be obtained by fitting the signal attenuation to some form of the StejskalTanner equation for unrestricted diffusion [? ? ]:

$$
S(g)=S_{0} e^{-D \gamma^{2} \delta^{2} g^{2} \Delta^{\prime}}
$$

where $S$ is the amplitude of a resonance, $S_{0}$ is the amplitude in the absence of diffusion, $D$ is the diffusion coefficient, $\gamma$ is the magnetogyric ratio, $g$ is the gradient strength, $\Delta^{\prime}$ is the corrected diffusion time and $\delta$ is the gradient pulse width.

The use of varying PFG amplitudes introduces a second dimension ("the diffusion dimension") to the NMR experiment. For a multicomponent solution in which signals from different components overlap in the NMR

*tel: 1-(949)-8245509, e-mail: mandelsh@uci.edu spectrum, the signals will in general undergo multiexponential decay along the diffusion dimension. This leads us to the inverse problem, which requires the evaluation of a Fourier transform (FT) along the acquisition dimension, followed by an inverse Laplace transform (LT) along the diffusion dimension. The $2 \mathrm{D}$ inverse problem can be simplified when factored into two 1D problems. One can first apply the FT to the free induction decay (FID) for each value of PFG amplitude. The resulting $2 \mathrm{D}$ data set can then in principle be analyzed by applying an inverse LT (or often, in practice, performing a multiexponential fit) along the diffusion dimension at each value of the chemical shift. In the simplest case, each resonance is generated by a single component of the mixture, hence a monoexponential fit suffices. This is the assumption used in HR-DOSY [?]).

Where there is no overlap between multiple peaks at a given chemical shift, a monoexponential fit can resolve compounds whose diffusion coefficients differ by as little as $1 \%$. Where the assumption of non-overlapping peaks is not satisfied, however, the result of a monoexponential fit is predictable but incorrect, producing a diffusion coefficient that is typically a compromise between the true values for the overlapping peaks. While a skilled spectroscopist can often interpret such a sub-optimal HR-DOSY plot if overlap is not too extensive, there is a pressing need for alternative analysis methods appropriate to the problem studied. In contrast to the case of exponential fitting, the solution to a multiexponential fit, while often useful [? ] with high-quality experimental data, is in general highly unstable. In particular, the results are 
method-dependent even in the case of a biexponential fit of noisy data, while higher exponential fits quickly become impossible. This is a notoriously difficult numerical problem that generally has no satisfactory solution (see e.g. ref. [? ]).

Because all resonances from a single compound are (with the notable exception of exchanging systems) expected to show a decay governed by a single diffusion coefficient, it can be advantageous to exploit this covariance and fit the entire spectrum instead of the individual peaks[? ? ? ? ? ]. Such multivariate methods are particularly useful for spectra, or sections of spectrum, containing signals of relatively few (2-3) species, but require at least $30 \%$ difference in diffusion coefficient between components and are more sensitive to systematic errors in experimental data than univariate methods such as HR-DOSY. The strengths of HR-DOSY and multivariate methods can be combined in hybrid analysis methods such as RECORD and LOCODOSY, in which smaller windows containing a limited number of components are subjected to separate multivariate processing and then combined [? ? ]. A number of the algorithms discussed above [? ? ? ? ? ? ? ? ? ? ? ] are currently implemented in the freely available DOSY Toolbox [? ].

The Filter Diagonalization Method (FDM) (see e.g. refs. [? ? ] and the review [? ] with references therein) avoids factorization, and instead attacks the $2 \mathrm{D}$ inverse problem directly. It essentially assumes, in line with other so-called "autoregression" methods, that the experimental data can be decomposed into a finite sum of stationary complex oscillations. The task of identifying the frequencies and amplitudes of these oscillations can be reduced to generalized eigenvalue problems. More precisely, in FDM the spectral analysis is performed locally by splitting the whole spectral range into small windows, and thus considering a series of small generalized eigenvalue problems. This treatment, using linear algebra, carries the benefit of improved computational efficiency as compared to nonlinear global optimization. While FDM has been previously used to analyze oscillatory NMR signals, its application to PFG NMR data is novel. A closely related approach, the Regularized Resolvent Transform (RRT), or more precisely a variant thereof (iRRT) that is adapted to the problem involving the inverse LT, has previously been applied to PFG NMR data [? ? ] with promising results. However, rather than solving generalized eigenvalue problems, RRT recasts the task of spectral estimation as a large number of linear least squares problems, making its computation many times more expensive than that of FDM.

The original argument of ref. [? ] in favor of iRRT was that the ill-posed nature of the inverse LT problem calls for a robust and reliable regularization method. While this is straightforward to implement for the linear least squares problem that appears in iRRT, no general robust and reliable algorithm seemed to be available for an illconditioned generalized eigenvalue problem. In fact the 2D FDM using the regularization scheme of ref. [?] gave results inferior to those obtained by iRRT. Here we revisit this problem, and demonstrate that a different regularization technique works, and produces spectra that are at least as good as iRRT DOSY spectra. Moreover, the fact that FDM estimates the spectra parametrically allows us to consider a wider variety of spectral representations than are available with iRRT spectra. In what follows, we describe an adaptation of 2D FDM for the DOSY analysis of 2D PFG NMR data. Both FDM and iRRT analysis have been incorporated into the DOSY toolbox [?].

\section{THEORY}

\section{The problems of harmonic inversion and spectral estimation}

We assume that in a DOSY experiment, PFG spectra are recorded for PFG amplitudes $\left(g_{m}\right)^{2} \propto m(m=$ $0,1,2, \ldots)$ such that the signal decays in the diffusion dimension exponentially as a function of $m$. A FID is then acquired for each value of $g_{m}$, resulting in a $2 \mathrm{D}$ data set $c_{n m}:=c\left(n \tau ; g_{m}\right)$ with $n=0, . ., N-1$ and $m=0, \ldots, M-1$, where index $n$ runs along the acquisition dimension sampled evenly with time increment $\tau$. (For simplicity such a dataset is referred to in what follows as a "2D time signal", although the actual dimensions are time and gradient amplitude). The DOSY signal can be parametrized as

$$
c_{n m}=\sum_{k=1}^{K} d_{k} u_{k}^{n} \lambda_{k}^{m}
$$

where

$$
u_{k}:=e^{-i \tau \omega_{k}} ; \quad \lambda_{k}:=e^{-\eta \alpha_{k}}
$$

in which the $\omega_{k}$ are the usual "complex frequencies" associated with the proton chemical shifts, and the $\alpha_{k}$ are proportional to the diffusion coefficients of the molecules associated with the peaks. The constant $\eta$ depends on the increment in field gradient amplitude and the experiment timing, as described by Eq. (??).

Physically one expects that $\lambda_{k}$ in the 2D harmonic inversion problem (??) are purely real (i.e., the time signal $c_{n m}$ does not oscillate in $m$ ) and both sets of poles satisfy $\left|u_{k}\right|<1$ and $\left|\lambda_{k}\right|<1$ (i.e., $c_{n m}$ decays in both $n$ and $m)$. However, neither of these constraints are explicitly imposed in FDM as they would prevent the harmonic inversion problem from having a linear algebraic solution. Thus, numerically both sets of poles are allowed to be any complex numbers with $u_{k}$ being spread preferably over the interior region of the unit disk close to the unit circle, and $\lambda_{k}$ concentrated around the real number line between 0 and 1 .

Define a 2D DOSY spectrum of $c_{n m}$ parametrized ac- 
cording to Eq. (??):

$$
I(\omega, \alpha)=\sum_{k=1}^{K} d_{k}\left(\frac{\tau}{1-u_{k} / u}\right)\left(\frac{\eta}{1-\lambda_{k} / \lambda}\right)
$$

with $u:=e^{-i \tau \omega}$ and $\lambda:=e^{-\eta \alpha}$. This spectral representation corresponds to a FT of $c_{n m}$ in the direct dimension followed by an inverse LT in the diffusion dimension. While $I(\omega, \alpha)$ should capture the relevant spectral features, the presence of imaginary components of $\alpha_{k}$ causes it to suffer from undesirable "tails", which are especially pronounced in the diffusion dimension, and which can make a plot of $I(\omega, \alpha)$ difficult to interpret, particularly when displayed using low contours. As suggested earlier [? ], such tails could be reduced by switching to pseudoabsorption lineshapes:

$$
I^{(2)}(\omega, \alpha)=\sum_{k=1}^{K} d_{k}\left(\frac{\tau}{1-u_{k} / u}\right)^{2}\left(\frac{\eta}{1-\lambda_{k} / \lambda}\right)^{2}
$$

Unfortunately, compared to a true double-absorption spectral representation, this representation distorts the relative peak amplitudes: each peak is scaled by a factor, $\left(\operatorname{Im} \alpha_{k} \operatorname{Im} \omega_{k}\right)^{-1}$, inversely proportional to the product of its widths in both dimensions, so that narrow peaks are overemphasized relative to broad ones.

At first glance, there would seem to be complete freedom to choose a desirable lineshape by manipulating the spectral parameters that appear in Eq. (??), for example replacing Lorentzian lineshapes with Gaussian. However, this can be done only using a stable, well-defined line list $\left\{d_{k}, u_{k}, \lambda_{k}, k=1, \ldots, K\right\}$, where each entry corresponds to an isolated peak. For realistic DOSY data such a line list is hardly feasible to obtain, thus limiting options severely. We therefore consider a "non-aggressive" modification of Eq. (??), in which Gaussian weighting functions

$$
\zeta^{\sigma}\left(\alpha-\alpha_{k}\right)=\exp \left[-\frac{\left(\alpha-\operatorname{Re} \alpha_{k}\right)^{2}}{2 \sigma^{2}}\right],
$$

are applied to eliminate the slowly-decaying tails in the diffusion dimension:

$$
I^{\sigma}(\omega, \alpha)=\sum_{k=1}^{K} d_{k}\left(\frac{\tau}{1-u_{k} / u}\right)\left(\frac{\eta \zeta^{\sigma}\left(\alpha-\alpha_{k}\right)}{1-\lambda_{k} / \lambda}\right)
$$

It is also useful to specify minimum widths for peaks in the chemical shift dimension $\left(\Gamma_{\omega}\right)$ and in the diffusion dimension $\left(\Gamma_{\alpha}\right)$. This effectively sets an upper bound on the resolving power of the method, preventing small numerical errors in the estimation of the imaginary parts of $\omega_{k}$ or $\alpha_{k}$ from giving physically unrealistic linewidths. Applying the transformations

$$
\begin{aligned}
& \omega_{k} \rightarrow \operatorname{Re}\left[\omega_{k}\right]+\operatorname{sign}\left(\operatorname{Im}\left[\omega_{k}\right]\right) i \Gamma_{\omega}, \text { if }\left|\operatorname{Im}\left[\omega_{k}\right]\right|<\Gamma_{\omega} \\
& \alpha_{k} \rightarrow \operatorname{Re}\left[\alpha_{k}\right]+\operatorname{sign}\left(\operatorname{Im}\left[\alpha_{k}\right]\right) i \Gamma_{\alpha}, \text { if }\left|\operatorname{Im}\left[\alpha_{k}\right]\right|<\Gamma_{\alpha}
\end{aligned}
$$

smooths the spectrum slightly and prevents erroneous peaks with low amplitudes but very low linewidths obtruding. By broadening narrow features, the spectrum is smoothed so as not to overemphasize spurious detail. Peak broadening also reduces the impact of digitization on the appearance of contour plots.

\section{The Filter Diagonalization Method}

Although in the original formulation of 2D FDM [? ] the parametric fit (??) was stated as the main objective, it was realized later that for noisy data, the $2 \mathrm{D}$ harmonic inversion problem, and especially the one associated with DOSY data, is extremely ill-posed. Here we utilize the version of the 2D FDM that only uses the parametrization (??) implicitly (i.e., a construction of a full coupled line list $\left\{d_{k}, u_{k}, \lambda_{k}, k=1, \ldots, K\right\}$ is avoided), but instead only solves a 2D spectral estimation problem [? ], i.e., estimating $I(\omega, \alpha)$ (or $I^{(2)}(\omega, \alpha)$ or $I^{\sigma}(\omega, \alpha)$ ) from a finite data set $\left\{c_{n m}\right\}$. The two problems are certainly related but the spectral estimation problem is much less demanding as it does not need to assume that the individual entries $\left(u_{k}, \lambda_{k}, d_{k}\right)$ are physically meaningful (or even available). Consequently, in what follows we will focus on the problem of spectral estimation. A brief derivation of 2D FDM will be provided; for more details, see the review [?].

The 1D FDM ansatz of ref. [? ], which relates the time signal to a quantum time autocorrelation function, is extended to the $2 \mathrm{D}$ case. We assume that the $2 \mathrm{D}$ time signal $c_{n m}$ can be written in the form [?]

$$
c_{n m}=\Phi^{\mathrm{T}} \hat{U}^{n} \hat{\Lambda}^{m} \Phi
$$

where $\Phi$ is a state vector ("a wavepacket") and $\hat{U}$ and $\hat{\Lambda}$ are commuting ("evolution") operators, i.e.

$$
\hat{U} \hat{\Lambda}=\Lambda \hat{U}
$$

These operators are also assumed to be complex symmetric (not to be confused with Hermitian); that is to say, for any two state vectors $\Psi$ and $\Phi$ the following identities are satisfied:

$(\Psi \mid \Phi)=(\Phi \mid \Psi) ;(\Psi|\hat{U}| \Phi)=(\Phi|\hat{U}| \Psi) ;(\Psi|\hat{\Lambda}| \Phi)=(\Phi|\hat{\Lambda}| \Psi)$,

where $(\Psi \mid \Phi)$, etc. define a complex symmetric (nonHermitian) inner product. $\hat{U}, \hat{\Lambda}$ and $\Phi$ in Eq. (??) are defined implicitly and are used to recast the harmonic inversion (??) as a linear algebra problem. Consider the eigenvalue problems for $\hat{U}$ and $\hat{\Lambda}$

$$
\hat{U} \Upsilon_{k}=u_{k} \Upsilon_{k} ; \quad \hat{\Lambda} \Upsilon_{k}=\lambda_{k} \Upsilon_{k}
$$

with the eigenvectors $\left\{\Upsilon_{k}\right\}$ forming an orthonormal set

$$
\left(\Upsilon_{k} \mid \Upsilon_{m}\right)=\delta_{k m}
$$


(Since $\hat{U}$ and $\hat{\Lambda}$ commute they possess a simultaneous eigenbasis.)

Substituting the spectral representations of $\hat{U}$ and $\hat{\Lambda}$

$$
\left.\hat{U}=\sum_{k} u_{k} \mid \Upsilon_{k}\right)\left(\Upsilon_{k}\left|; \quad \hat{\Lambda}=\sum_{k} \lambda_{k}\right| \Upsilon_{k}\right)\left(\Upsilon_{k} \mid\right.
$$

into Eq. (??) we obtain Eq. (??) with

$$
d_{k}=\left(\Upsilon_{k} \mid \Phi\right)^{2}
$$

That is, the two assumptions (??) and (??) are equivalent. The ansatz of Eq. (??) allows us to rewrite the parametric spectral representations (??) and (??) using an alternative form:

$$
\begin{aligned}
I(\omega, \alpha) & =\left(\Phi\left|\left(\frac{\tau}{1-\hat{U} / u}\right)\left(\frac{\eta}{1-\hat{\Lambda} / \lambda}\right)\right| \Phi\right) \\
I^{(2)}(\omega, \alpha) & =\left(\Phi\left|\left(\frac{\tau}{1-\hat{U} / u}\right)^{2}\left(\frac{\eta}{1-\hat{\Lambda} / \lambda}\right)^{2}\right| \Phi\right)(
\end{aligned}
$$

Next, we wish to convert the operator eigenvalue problems (??) into matrix eigenvalue problems, so that they can be solved numerically. This requires suitable matrix representations of the operators $\hat{U}$ and $\hat{\Lambda}$, which so far have been defined only implicitly. To this end, consider a basis $\left\{\Psi_{j}\right\}$, which is a Fourier basis in the direct (chemical shift) dimension:

$$
\begin{aligned}
& \Psi_{j}=\sum_{m=0}^{\tilde{M}} \sum_{n=0}^{\tilde{N}}\left(\hat{U} / z_{j}\right)^{n} \hat{\Lambda}^{m} \Phi \\
& \text { with } z_{j}:=e^{-i \tau \varphi_{j}}
\end{aligned}
$$

where $\tilde{N}=N / 2$ and $\tilde{M}=M / 2$, assuming for simplicity that both $N$ and $M$ are even integers, and $\left\{\varphi_{j}, j=1, \ldots, K_{\text {win }}\right\}$ is an evenly spaced grid with spacing $\Delta \varphi \leq 2 \pi /(N \tau)$ that spans a window in the frequency domain, $\varphi_{j} \in\left[\omega^{\min } ; \omega^{\max }\right]$. Just for now, we can assume that this window coincides with the entire Nyquist range, $[-\pi / \tau ; \pi / \tau]$. It will be shown that a small window in the frequency domain could suffice. In principle, an additional Fourier basis transformation could be performed in the second dimension, but because of the lack of oscillations in the diffusion dimension, such a basis would be highly ill-conditioned (linearly-dependent) and would provide little benefit.

We assume that the eigenvectors $\Upsilon_{k}$ can be well represented using the Fourier basis:

$$
\Upsilon_{k}=\sum_{j} \mathbf{B}_{k j} \Psi_{j}
$$

where we have introduced a column vector $\mathbf{B}_{k}=$ $\left(\mathbf{B}_{k 1}, \ldots, \mathbf{B}_{k K_{\text {win }}}\right)^{\mathrm{T}}$ containing the expansion coefficients.

Define the following $K_{\text {win }} \times K_{\text {win }}$ matrices, which are respectively the overlap matrix and the matrix representations of operators $\hat{U}$ and $\hat{\Lambda}$ in this basis:

$$
\begin{aligned}
& {\left[\mathbf{U}_{0}\right]_{j j^{\prime}}=\left(\Psi_{j} \mid \Psi_{j^{\prime}}\right)} \\
& {\left[\mathbf{U}_{1}\right]_{j j^{\prime}}=\left(\Psi_{j}|\hat{U}| \Psi_{j^{\prime}}\right)} \\
& {\left[\mathbf{U}_{2}\right]_{j j^{\prime}}=\left(\Psi_{j}|\hat{\Lambda}| \Psi_{j^{\prime}}\right)}
\end{aligned}
$$

These matrices can be evaluated explicitly in terms of the available data $\left\{c_{n m}\right\}$ by substituting Eq. (??) into Eq. (??) and than utilizing Eq. (??):

$$
\begin{aligned}
& {\left[\mathbf{U}_{0}\right]_{j j^{\prime}}=\sum_{m=0}^{\tilde{M}} \sum_{n=0}^{\tilde{N}} \sum_{m^{\prime}=0}^{\tilde{M}} \sum_{n^{\prime}=0}^{\tilde{N}} c_{n+n^{\prime}, m+m^{\prime}}\left(z_{j}\right)^{-n}\left(z_{j^{\prime}}\right)^{-n^{\prime}}} \\
& {\left[\mathbf{U}_{1}\right]_{j j^{\prime}}=\sum_{m=0}^{\tilde{M}} \sum_{n=0}^{\tilde{N}} \sum_{m^{\prime}=0}^{\tilde{M}} \sum_{n^{\prime}=0}^{\tilde{N}} c_{n+n^{\prime}+1, m+m^{\prime}}\left(z_{j}\right)^{-n}\left(z_{j^{\prime}}\right)^{-n^{\prime}}} \\
& {\left[\mathbf{U}_{2}\right]_{j j^{\prime}}=\sum_{m=0}^{\tilde{M}} \sum_{n=0}^{\tilde{N}} \sum_{m^{\prime}=0}^{\tilde{M}} \sum_{n^{\prime}=0}^{\tilde{N}} c_{n+n^{\prime}, m+m^{\prime}+1}\left(z_{j}\right)^{-n}\left(z_{j^{\prime}}\right)^{-n^{\prime}}}
\end{aligned}
$$

Though correct, each of the above expressions includes a summation over 4 indices, which is numerically very expensive. Substantially more efficient expressions are given in the next section.

Substituting Eq. (??) into Eq. (??) we obtain generalized eigenvalue problems

$$
\mathbf{U}_{1} \mathbf{B}_{k}=u_{k} \mathbf{U}_{0} \mathbf{B}_{k} ; \quad \mathbf{U}_{2} \mathbf{B}_{k}=\lambda_{k} \mathbf{U}_{0} \mathbf{B}_{k}
$$

Note that the eigenvectors are mutually orthogonal:

$$
\mathbf{B}_{k^{\prime}}^{\mathrm{T}} \mathbf{U}_{0} \mathbf{B}_{k}=0 \quad\left(k \neq k^{\prime}\right)
$$

In addition, we normalize them according to

$$
\mathbf{B}_{k}^{\mathrm{T}} \mathbf{U}_{0} \mathbf{B}_{k}=1
$$

Solving Eq. (??), in principle, provides the solution of the harmonic inversion problem (??), but unless the data $\left\{c_{n m}\right\}$ being processed fit exactly the form of Eq. (??), there is a hidden problem that still remains: numerically, the two eigenvalue problems in Eq. (??) result in two distinct sets of eigenvectors $\left\{\mathbf{B}_{k}\right\}$ and $\left\{\mathbf{A}_{k^{\prime}}\right\}$ that are generally impossible to couple in a meaningful way. Therefore, we instead solve two independent generalized eigenvalue problems:

$$
\mathbf{U}_{1} \mathbf{B}_{k}=u_{k} \mathbf{U}_{0} \mathbf{B}_{k} ; \quad \mathbf{U}_{2} \mathbf{A}_{k^{\prime}}=\lambda_{k^{\prime}} \mathbf{U}_{0} \mathbf{A}_{k^{\prime}}
$$

with normalization conditions

$$
\mathbf{B}_{k}^{\mathrm{T}} \mathbf{U}_{0} \mathbf{B}_{k}=1 ; \quad \mathbf{A}_{k^{\prime}}^{\mathrm{T}} \mathbf{U}_{0} \mathbf{A}_{k^{\prime}}=1
$$

Although with two uncoupled sets of eigenvectors a complete solution of the harmonic inversion problem is impossible, ref. [? ] shows how, in spite of this difficulty, 
a meaningful 2D spectrum can still be constructed. First, define the cross correlation matrix

$$
\mathbf{D}_{k k^{\prime}}=\mathbf{B}_{k}^{\mathrm{T}} \mathbf{U}_{0} \mathbf{A}_{k^{\prime}}
$$

Ideally, one wants the two sets of eigenvectors, $\left\{\mathbf{B}_{k}\right\}$ and $\left\{\mathbf{A}_{k^{\prime}}\right\}$, to be identical, generating a matrix $\mathbf{D}$ whose rows could be reordered to form a unit matrix. Generally, the two sets of eigenvectors are distinct, leading to a full D matrix with some elements $\mathbf{D}_{k k^{\prime}} \sim 1$ (corresponding to nearly simultaneous eigenvectors) and most other elements, very small.

Define the column vectors $\mathbf{b}(\omega), \mathbf{b}^{(2)}(\omega), \mathbf{a}(\alpha), \mathbf{a}^{(2)}(\alpha)$ and $\mathbf{a}^{\sigma}(\alpha)$, with components

$$
\begin{aligned}
\mathbf{b}_{k}(\omega) & =\left(\frac{\tau}{1-u_{k} / u}\right) \mathbf{B}_{k}^{\mathrm{T}} \mathbf{C} \\
\mathbf{b}_{k}^{(2)}(\omega) & =\left(\frac{\tau}{1-u_{k} / u}\right)^{2} \mathbf{B}_{k}^{\mathrm{T}} \mathbf{C} \\
\mathbf{a}_{k}(\alpha) & =\left(\frac{\eta}{1-\lambda_{k} / \lambda}\right) \mathbf{A}_{k}^{\mathrm{T}} \mathbf{C} \\
\mathbf{a}_{k}^{(2)}(\alpha) & =\left(\frac{\eta}{1-\lambda_{k^{\prime}} / \lambda}\right)^{2} \mathbf{A}_{k}^{\mathrm{T}} \mathbf{C} \\
\mathbf{a}_{k}^{\sigma}(\alpha) & =\left(\frac{\eta \zeta^{\sigma}\left(\alpha-\alpha_{k}\right)}{1-\lambda_{k} / \lambda}\right) \mathbf{A}_{k}^{\mathrm{T}} \mathbf{C}
\end{aligned}
$$

where the elements of the data array $\mathbf{C}=$ $\left(f_{1}^{(0)}, \ldots, f_{K_{\text {win }}}^{(0)}\right)^{\mathrm{T}}$ are defined in the next section (Eq. (??)).

The three spectral representations can now be written as:

$$
\begin{aligned}
I(\omega, \alpha) & =\mathbf{b}(\omega)^{\mathrm{T}} \mathbf{D} \mathbf{a}(\alpha) \\
I^{(2)}(\omega, \alpha) & =\mathbf{b}^{(2)}(\omega)^{\mathrm{T}} \mathbf{D} \mathbf{a}^{(2)}(\alpha) \\
I^{\sigma}(\omega, \alpha) & =\mathbf{b}(\omega)^{\mathrm{T}} \mathbf{D} \mathbf{a}^{\sigma}(\alpha)
\end{aligned}
$$

\section{Working expressions for evaluating the data matrices}

The expressions for the data matrices $\mathbf{U}_{p}(p=0,1,2)$ introduced in Eq. (??) can be simplified, evaluating the sums over $m, m^{\prime}$ and $n^{\prime}$. Define the arrays

$$
\begin{aligned}
f_{j}^{(p)} & :=\sum_{n=0}^{\tilde{N}-1} a_{n}^{(p)} z_{j}^{-n}, \\
g_{j}^{(p)} & :=\sum_{n=0}^{\tilde{N}-2} a_{n+\tilde{N}}^{(p)} z_{j}^{-n}
\end{aligned}
$$

with

$$
\begin{aligned}
& a_{n}^{(0)}:=\sum_{m=0}^{M-2} c_{n, m}(\tilde{M}-|\tilde{M}-m-1|), \\
& a_{n}^{(1)}:=\sum_{m=0}^{M-2} c_{n+1, m}(\tilde{M}-|\tilde{M}-m-1|), \\
& a_{n}^{(2)}:=\sum_{m=0}^{M-2} c_{n, m+1}(\tilde{M}-|\tilde{M}-m-1|)
\end{aligned}
$$

For the off-diagonal elements $\left(j \neq j^{\prime}\right)$, we have [? ]

$$
\left[\mathbf{U}_{p}\right]_{j j^{\prime}}=\frac{z_{j}\left(f_{j^{\prime}}^{(p)}-z_{j}^{-M} g_{j^{\prime}}^{(p)}\right)-z_{j^{\prime}}\left(f_{j}^{(p)}-z_{j^{\prime}}^{-M} g_{j}^{(p)}\right)}{z_{j}-z_{j^{\prime}}}
$$

and for the diagonal elements,

$$
\left[\mathbf{U}_{p}\right]_{j j}=\sum_{n=0}^{N-2}(\tilde{N}-|\tilde{N}-1-n|) a_{n}^{(p)} z_{j}^{-n}
$$

Numerical evaluation of equations (??)-(??) is already very inexpensive, but only the sums for $p=0$ have to be computed explicitly; for $p>0$ one can use recursive formulae. Moreover, with a suitable choice for the grid $\left\{\varphi_{j}, j=1, \ldots, K_{\text {win }}\right\}$ (note that $z_{j}=e^{-i \tau \varphi_{j}}$ ), the above summations can be evaluated using a fast FT.

\section{Numerical solution of the generalized eigenvalue problems: windowing and regularization}

The solution of the generalized eigenvalue problems (??) involving $K_{\text {win }} \times K_{\text {win }}$ square matrices scales as $\sim K_{w i n}^{3}$, which for large data sets (large $N$ ) may become unacceptably expensive if a large window with many basis functions is used. However, due to the use of the Fourier basis (??), the structure of $\mathbf{U}$ is such that very accurate eigenpairs $\left(u_{k}, \mathbf{B}_{k}\right)$ and $\left(\lambda_{k^{\prime}}, \mathbf{A}_{k^{\prime}}\right)$ can be obtained by solving these equations in block-diagonal fashion, i.e., by splitting the Nyquist range into a number of small windows $\left[\omega_{l}^{\min } ; \omega_{l}^{\max }\right]$ and considering only the corresponding small $\left(K_{w i n} \times K_{w i n}\right)$ block for each window.

Another key issue in the 2D FDM is that the generalized eigenvalue problems in Eq. (??) are typically very ill-conditioned, so that their exact numerical solution, when used in e.g. Eq. (??), yields spectra that are contaminated by artifacts. This, consequently, calls for regularization of the data matrices. Our numerical tests indicate that the previously proposed [? ] regularization scheme named "FDM2k" gives poor results. In FDM2k the original generalized eigenvalue problems (??) are replaced by

$$
\begin{aligned}
\mathbf{U}_{0}^{\dagger} \mathbf{U}_{1} \mathbf{B}_{k} & =u_{k}\left(\mathbf{U}_{0}^{\dagger} \mathbf{U}_{0}+q^{2} \mathbf{I}\right) \mathbf{B}_{k} \\
\mathbf{U}_{0}^{\dagger} \mathbf{U}_{2} \mathbf{A}_{k^{\prime}} & =\lambda_{k^{\prime}}\left(\mathbf{U}_{0}^{\dagger} \mathbf{U}_{0}+q^{2} \mathbf{I}\right) \mathbf{A}_{k^{\prime}}
\end{aligned}
$$


The degree of regularization, designated by the regularization parameter $q$, is normalized by including the scaling factor $\bar{\sigma}=\left\langle\left|\left[\mathbf{U}_{0}\right]_{j j}\right|\right\rangle$, which is set equal to the average value of the diagonal elements of $\mathbf{U}_{0}$. (A scaling factor that depends on the properties of $\mathbf{U}_{0}$ in a more sophisticated fashion could also be used, which would reduce the ambiguity in the choice of the regularization parameter $q$.

An alternative regularization can be implemented by using the singular value decomposition (SVD) of $\mathbf{U}_{0}$ :

$$
\mathbf{U}_{0}=\mathbf{V} \boldsymbol{\Sigma} \mathbf{W}^{\dagger}
$$

where $\mathbf{V} \mathbf{V}^{\dagger}=I, \mathbf{W} \mathbf{W}^{\dagger}=I$ and $\boldsymbol{\Sigma}=\operatorname{diag}\left\{\sigma_{k}\right\}$ with real $\sigma_{k}>0$. Now replacing

$$
\mathbf{U}_{0} \rightarrow \mathbf{V} \boldsymbol{\Sigma}_{q} \mathbf{W}^{\dagger} \quad \text { with } \quad \boldsymbol{\Sigma}_{q}=\operatorname{diag}\left\{\frac{\sigma_{k}^{2}+(q \bar{\sigma})^{2}}{\sigma_{k}}\right\}
$$

and solving Eq. (??) one achieves the result similar to that of Eq. (??), i.e. still poor.

However, in this work we discovered yet a new and embarrassingly simple regularization procedure based on replacing the diagonal matrix $\boldsymbol{\Sigma}$ with $\boldsymbol{\Sigma}_{q}$, in which all singular values $\sigma_{k}<q \bar{\sigma}$ are replaced with $q \bar{\sigma}$, where the latter plays the same role of a regularization parameter as before. This regularization turns out to be superior to that in Eq. (??) and to all the other regularization recipes that we have tested so far.

\section{iRRT: Regularized Resolvent Transform for DOSY processing}

Here for completeness we also include working expressions for the iRRT method, which is closely related to FDM conceptually, but differs in the numerical evaluation of the spectra (equations (??) and (??)) and, particularly, in how the regularization is implemented.

If the data $c_{n m}$ satisfy Eq. (??) (and, consequently, Eq. (??)) exactly, it can be shown that the FDM expressions for the spectra (??) and (??) can be rewritten in an equivalent form

$$
\begin{aligned}
I(\omega, \alpha) & =\tau \eta \mathbf{C}^{\mathrm{T}} \mathbf{R}_{1}^{-1} \mathbf{U}_{0} \mathbf{R}_{2}^{-1} \mathbf{C} \\
I^{(2)}(\omega, \alpha) & =\tau^{2} \eta^{2} \mathbf{C}^{\mathrm{T}} \mathbf{R}_{1}^{-1} \mathbf{U}_{0} \mathbf{R}_{1}^{-1} \mathbf{U}_{0} \mathbf{R}_{2}^{-1} \mathbf{U}_{0} \mathbf{R}_{2}^{-1} \mathbf{C}
\end{aligned}
$$

where we defined the two matrix pencils

$$
\mathbf{R}_{1}=\mathbf{U}_{0}-\mathbf{U}_{1} / u ; \quad \mathbf{R}_{2}=\mathbf{U}_{0}-\mathbf{U}_{2} / \lambda
$$

Generally, the matrices $\mathbf{R}_{1}$ and $\mathbf{R}_{2}$ are highly illconditioned, so that the use of their exact inverses leads to spectra with artifacts. In iRRT this problem is solved by using pseudo-inverses [? ]. For example, a pseudoinverse of $\mathbf{R}_{1}$ can be obtained by replacing it with its regularized matrix, as in the regularization of $\mathbf{U}_{0}$ in the previous section. That is, at each frequency $\omega$ the SVD of $\mathbf{R}_{1}$ is performed

$$
\mathbf{R}_{1}=\mathbf{W} \boldsymbol{\Sigma} \mathbf{V}^{\dagger}
$$

Given a regularization parameter $q>0$, a pseudo-inverse of $\mathbf{R}_{1}$ can then be computed by first replacing

$$
\sigma_{k} \rightarrow q \bar{\sigma}, \text { if } \sigma_{k}<q \bar{\sigma}
$$

and then using

$$
\mathbf{R}_{1}^{-1} \rightarrow \mathbf{V} \mathbf{\Sigma}_{q}^{-1} \mathbf{W}^{\dagger}
$$

where for the scaling factor we use the same expression as that defined for regularization of $\mathbf{U}_{0}$ in the previous section, namely, $\bar{\sigma}=\left\langle\left|\left[\mathbf{U}_{0}\right]_{j j}\right|\right\rangle$. The pseudoinverse of $\mathbf{R}_{2}$ is evaluated following the same procedure.

The implementation of iRRT for each frequency window requires a SVD of a $K_{w i n} \times K_{w i n}$ matrix $\mathbf{R}_{1}(\omega)$ to be performed for every value of $\omega$ used to represent the spectra, and then SVD of $\mathbf{R}_{2}(\alpha)$ for every value of $\alpha$. This is to be compared in the FDM with a single SVD of $\mathbf{U}_{0}$ followed by the solution of two generalized eigenvalue problems (??).

\section{EXPERIMENTAL}

The DOSY data from sample 1 used in this investigation were acquired using a sample containing dextran, tartrazine, ephedrine, TSP (sodium 3-(trimethylsilyl)propionate-2,2,3,3- $\mathrm{d}_{4}$ ), nicotinic acid and ethanol, in $\mathrm{D}_{2} \mathrm{O}$. For further details see the original paper on LOCODOSY[? ]. Sample 2 was made up in $80 \mathrm{mM}$ phosphate buffer at $7.4 \mathrm{pD}$, and contained $10 \mathrm{mM}$ TSP as a reference compound, with $3.75 \mathrm{mM}$ of the tripeptide Val-TyVal, $3.75 \mathrm{mM}$ of phenol, and $5.0 \mathrm{mM}$ of propan-1-ol.The DOSY data for sample 2 were acquired on a $500 \mathrm{MHz}$ Bruker DRX spectrometer equipped with a $53 \mathrm{G} \mathrm{cm}^{-1} 5$ mm probe using the Oneshot45 ([? ]) pulse sequence. 256 transients of 16378 complex points were acquired for each of 15 gradient increments ranging from 5.3 to 42.4 $\mathrm{G} \mathrm{cm}^{-1}$ nominal amplitude. Equal increments in gradient squared were used with half-sine shaped gradient pulses of $2.5 \mathrm{~ms}$ duration and with a diffusion delay of $100 \mathrm{~ms}$.

\section{RESULTS AND DISCUSSION}

A DOSY plot always needs interpretation. This can be straightforward (e.g. when spectra are well resolved), but frequently a critical examination of the data is necessary. In such cases it is important to compare the results of applying different processing algorithms. Here we compare the results obtained with the new method, FDMDOSY, to those obtained using the existing iRRT-DOSY together with three methods already implemented in the DOSYToolbox: the univariate methods, HRDOSY, and 


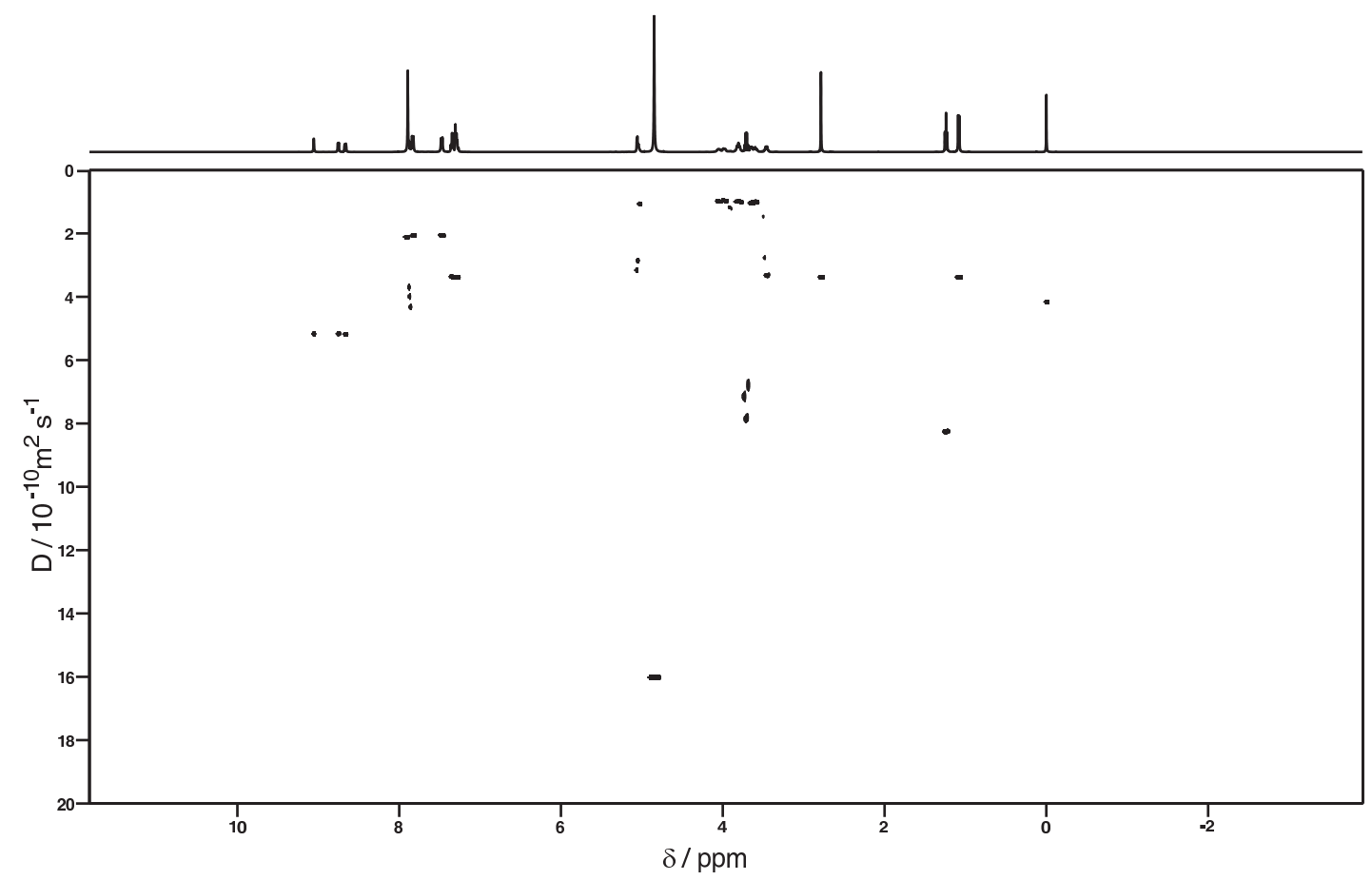

Figure 1: HR-DOSY plot of sample 1, containing seven molecular species (dextran, tartrazine, ephedrine, TSP, nicotinic acid and ethanol, in $\mathrm{D}_{2} \mathrm{O}$ ). Where signals overlap in the spectrum the apparent diffusion coefficients appear at compromise values.

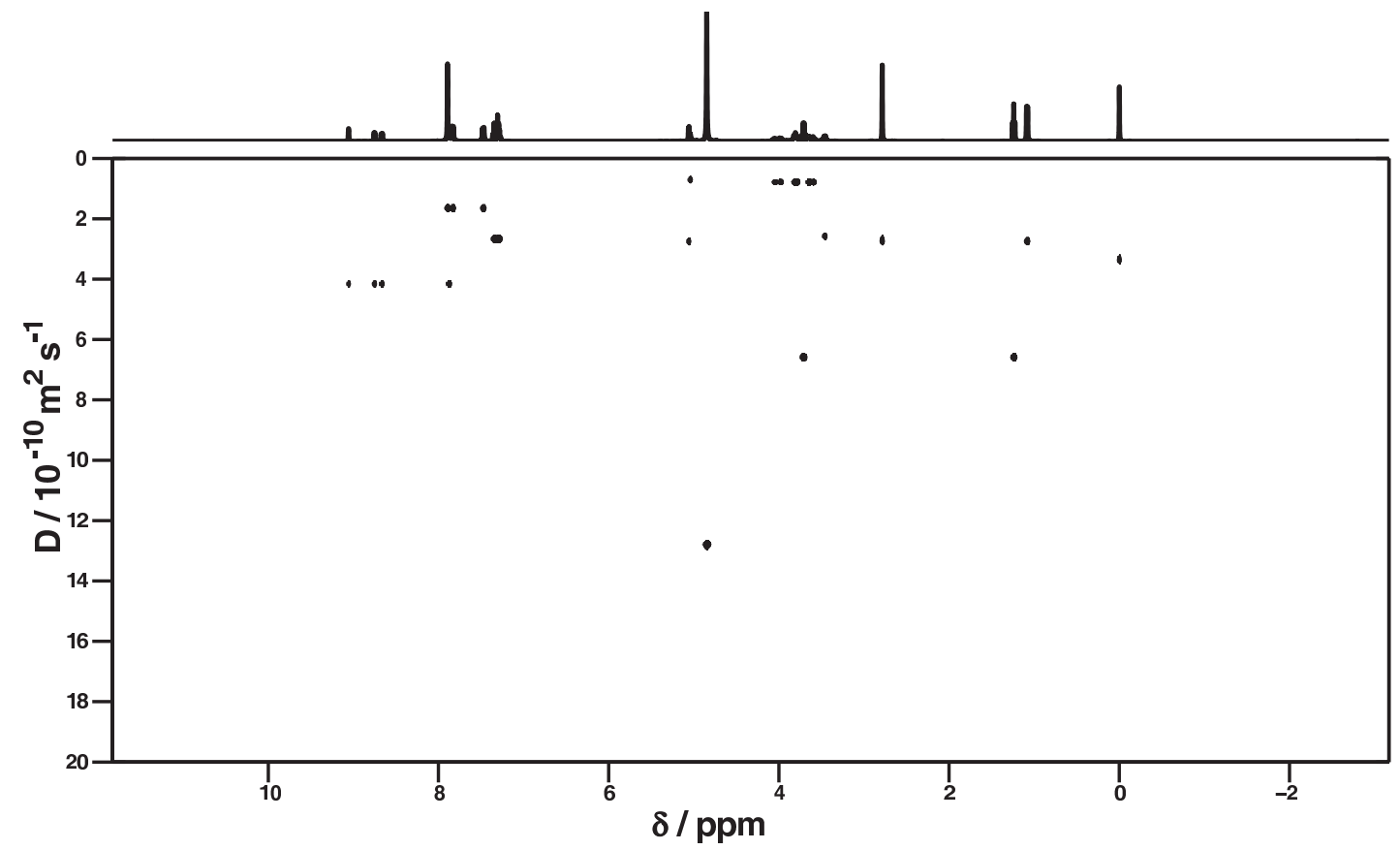

Figure 2: LOCODOSY plot of sample 1, containing seven molecular species. All signals appear at their correct apparent diffusion coefficient.

biexponential fitting, and the more advanced multivariate algorithm, LOCODOSY, which is better adapted to systems with overlapping peaks in the chemical shift dimension. HR-DOSY is quick and stable, and when the underlying assumption that each resonance belongs to a single species is violated it fails in a predictable way.

The HR-DOSY plot (Fig. ??) of a seven component mixture (sample 1) illustrates the method's strengths and weaknesses.

Most of the signals are well-resolved, and the calcu- 


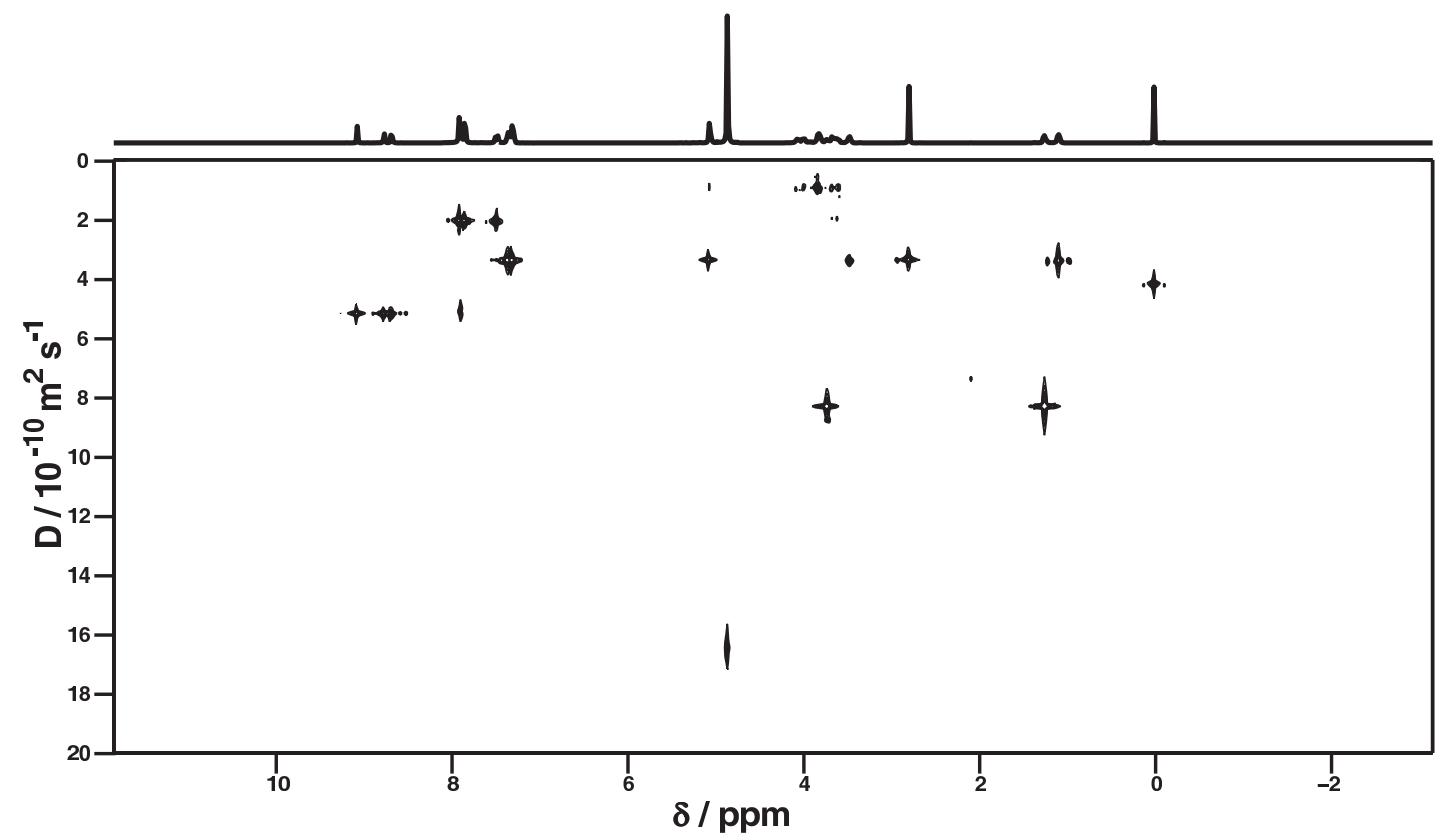

Figure 3: iRRT-DOSY pseudoabsorption mode plot of sample 1 , using $N=4096, M=30, K_{\text {win }}=200, q=10$. Signals appear at their correct apparent diffusion coefficient, but the peaks have an undesirable shape and distorted amplitudes due to the pseudoabsorption representation.

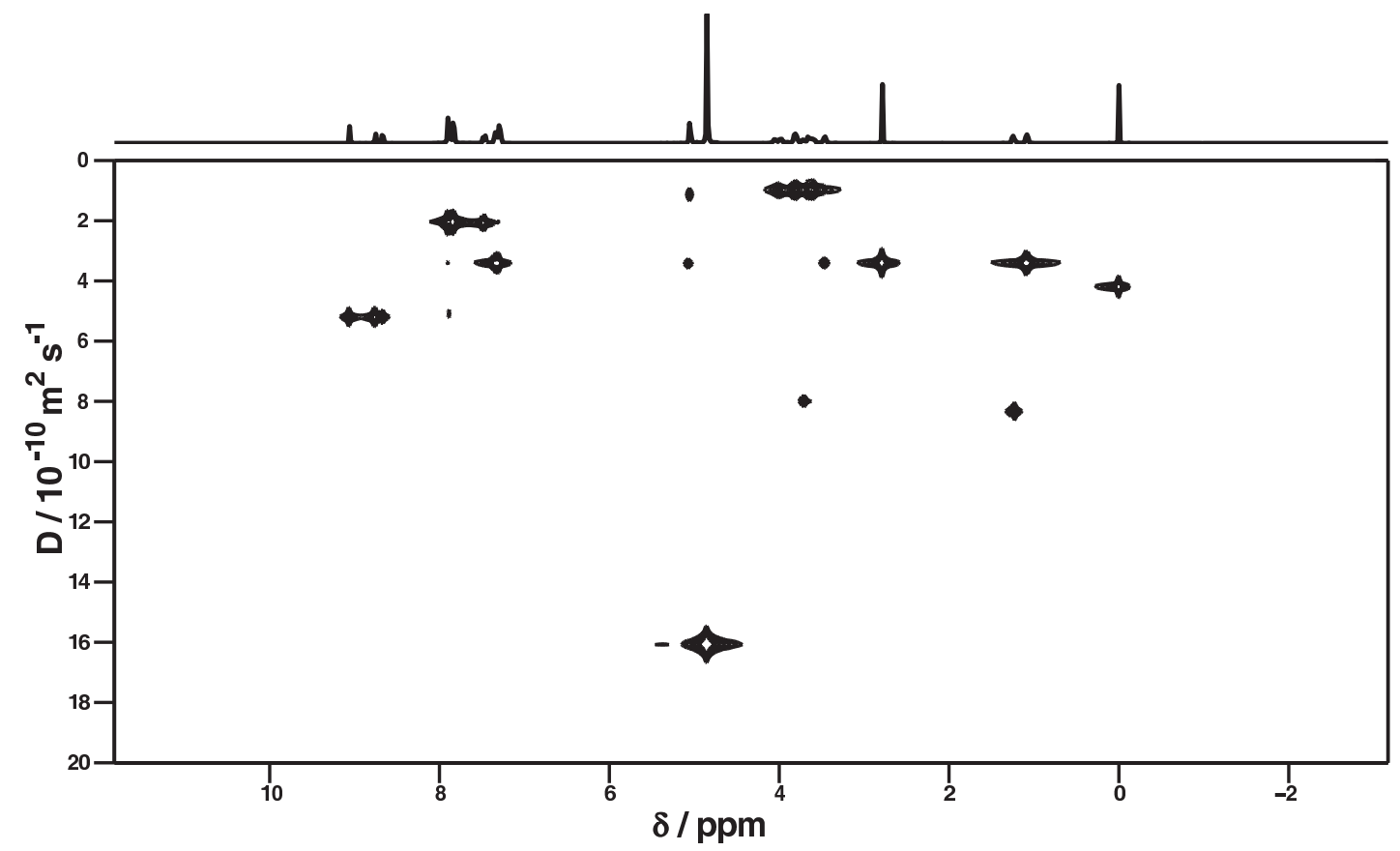

Figure 4: FDM-DOSY pseudoabsorption mode plot of sample $1 . \quad N=4096, M=30, K_{\text {win }}=200, q=1, \Gamma_{\omega}=0, \Gamma_{\alpha}=0$. Signals appear at their correct apparent diffusion coefficient. Just as in the RRT processing, the peaks have an undesirable shape and distorted amplitudes due to the pseudoabsorption representation.

lated diffusion coefficients are reliable, accurate and unambiguous. However, in a few places (e.g around 8 ppm, $5 \mathrm{ppm}$, and $3 \mathrm{ppm}$ ) resonances overlap and the diffusion coefficient is not well defined. Factorization of the 2D problem has led to each peak being assigned an apparent diffusion coefficient without regard to the possibility of overlap with nearby peaks. Where overlap does occur, incorrect diffusion coefficients are obtained and, for example, peaks within a given multiplet are no longer properly aligned. It is in such cases as these that other processing 


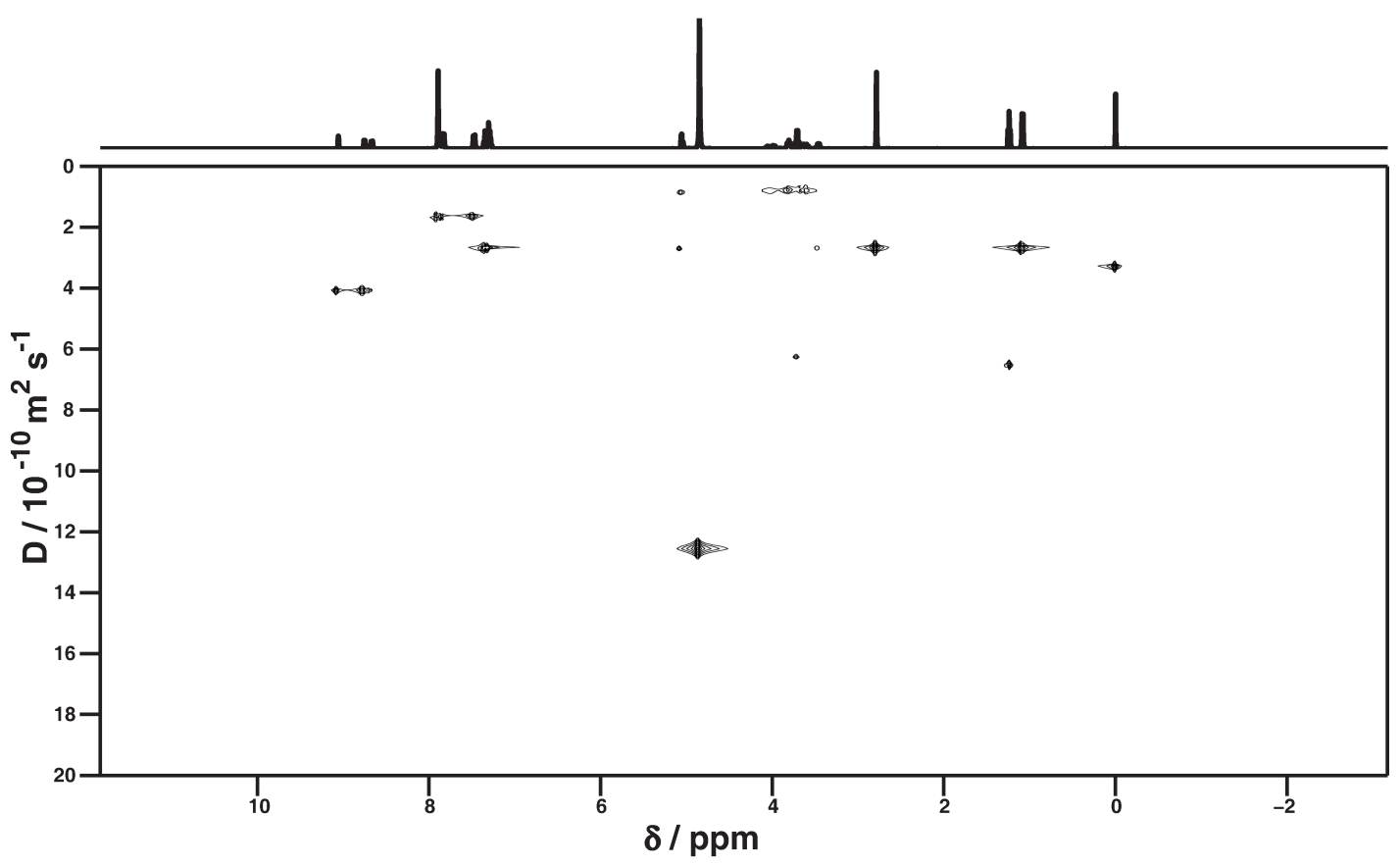

Figure 5: FDM-DOSY absolute value mode spectra with Gaussian masking for sample 1. $N=4096, M=30, K_{\text {win }}=200$, $q=1$ and $\sigma=0.2 \cdot 10^{-10} \mathrm{~m}^{2} \mathrm{~s}^{-1}, \Gamma_{\omega}=0, \Gamma_{\alpha}=0$. Signals appear at their correct apparent diffusion coefficient, with more faithful peak amplitudes due to the absolute value display; the peak appearance is greatly improved by Gaussian masking

methods can have advantage. One such method, LOCODOSY has been shown to produce a DOSY spectrum that corresponds very well to the known composition where only a small number of species contribute to the signal at any one chemical shift. LOCODOSY works by processing the spectrum piecemeal, using windows which span a small interval of chemical shift, each of which is assumed to contain peaks from only a few species. It can produce beautiful results (as in Fig. ??), but the big drawback is that for the best results intelligent user input is required when segmenting the spectrum, as there is no general way to automatically decide the window boundaries and the number of components within each window (the automatic determination of number of components works well in most cases and fails in a predictable way). In many cases, the spectrum can be auto-partitioned, only requiring the user to visually inspect the spectrum and assign a threshold value.

The closest relative of FDM-DOSY is iRRT-DOSY [? ], which has previously been shown to give good results. However, FDM possesses two advantages over iRRT: while the spectral windows and the data matrices involved in FDM are the same as those in iRRT, the FDM algorithm is significantly faster. iRRT runtime scales poorly with both the chosen matrix size, and with the image resolution of the rasterized DOSY plot. Hence iRRT may only be suitable for computing small regions of the full spectrum. Perhaps more importantly, FDM provides spectral parameters which allow a wider range of spectral representations to be used than is the case for the iRRT. For example, in iRRT one has essentially to choose between two spectral representations. Absolute value mode (??) has long tails in both dimensions which form a cross-hair shape, which the typical spectroscopist may find very unfamiliar. The double-absorption lineshape provided by the pseudoabsorbtion mode (??), has rapidly decaying tails so that when viewed with the correct contour levels, the peaks generated are more comparable to those produced by other methods. Unfortunately this means that the narrow peaks are overemphasized relative to the broad peaks. Fig. ?? shows the doubleabsorption iRRT spectrum of the same DOSY data as used above. The following processing parameters were used: $N=4096, K_{\text {win }}=200, \sigma_{\alpha}=0.2$, and $q=10$. While all the spectral features are present, the spectral appearance is certainly inferior, with distorted relative intensities. 

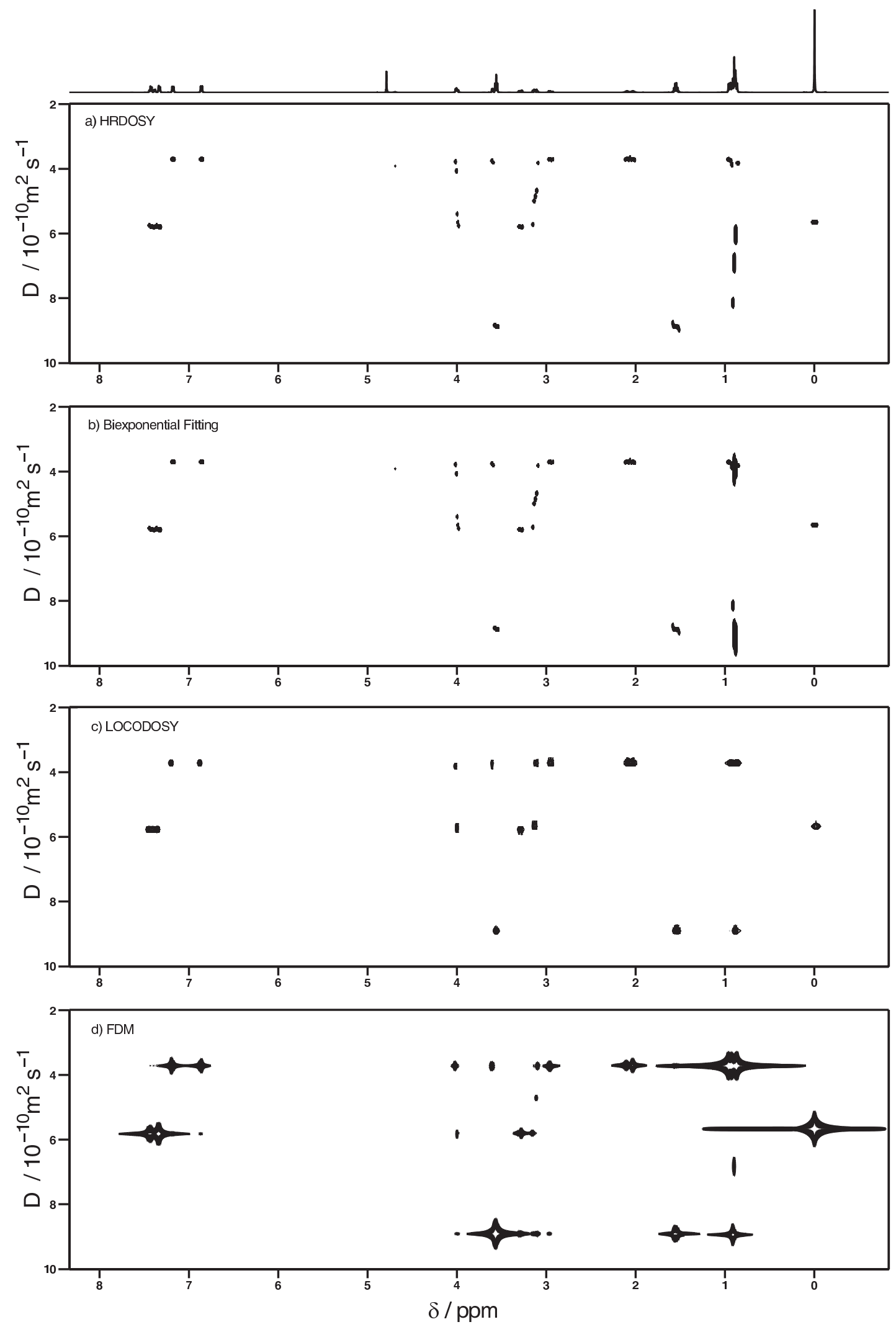

Figure 6: Comparison of four different processing methods for the DOSY data of sample 2 (a 4 component mixture of Val-TyrVal, phenol, propan-1-ol and TSP): a) HR-DOSY, b) biexponential fitting, c) LOCODOSY, and d) FDM-DOSY. 
FDM provides more flexibility for the spectral representation. As an example, using FDM one can correct the unwanted long tails of the peaks in the absolute value mode by Gaussian masking ( $c f$. Eq. (??)), and by setting a lower limit on the peak width ( $c f$. Eq. (??)) one can reduce the effect of amplitude distortion in the pseudoabsorption spectrum. This is demonstrated in Figures ?? and ??, in which we show the FDM absolute value spectrum and pseudo-absorption spectrum, respectively. (The FDM calculations used the same set of parameters as those for the iRRT, with the exception of regularization parameter $q$.)

While the peak list produced by FDM is independent of the choice of display mode, the choice of graphical representation is under user control. Depending on the primary goal, the optimal settings for creating a DOSY plot may differ depending on whether easy determination of diffusion coefficients or a generally visually pleasing spectrum is sought.

As a second example (Fig. ??), we demonstrate the various discussed processing methods on a 4-component mixture (the solvent, HOD, peak is excluded from analysis). There are overlapping signals at around 1, 3 and $4 \mathrm{ppm}$, which in the HR-DOSY spectrum causes peaks at those frequencies to have diffusion coefficients at values intermediate between those of the species involved. In the biexponential fitting, the overlap at $1 \mathrm{ppm}$ is resolved but for the other overlaps, at 3 and $4 \mathrm{ppm}$, the signal-to-noise ratio is insufficient for a biexpoential fit to resolve the overlap at the given diffusion coefficients. The FDM-DOSY approach is much more successful, with most signals in their correct positions. For this particular data set the best result is obtained with fully automated LOCODOSY processing. In this example it is clear that comparing different processing methods is a valuable aid in assigning the component NMR spectra.

\section{CONCLUSIONS}

We have compared the DOSY plots produced by the DOSY Toolbox [? ] using five different methods. Al- though the appearance of the plots varies, the features identified by each method are similar in each case.

HR-DOSY is quick (i.e. seconds) and stable, and the only parameters to set are the spectral threshold determining minimum peak height to be processed and the form of the decay (typically pure exponential, or compensated for non-uniformity of the field gradients present in some spectrometers [? ]).

The biexponential fit is dependent on starting values, so a large number of these have to be compared, typically making the process orders of magnitude slower.

FDM typically gives acceptable results with the default values, but can give small spurious peaks, so the regularization is under user control to allow different regularizations to be compared. An empirical value $q=0.7$ provided the best suppression of artifacts in the examples in this paper. In principle, one could further optimize the appearance of the spectral peaks by specifying minimum widths $\Gamma_{\alpha}$ and $\Gamma_{\omega}$ in the chemical shift and diffusion dimensions, while still resolving the components of the mixture. However, this feature introduces additional adjustable parameters in the FDM processing, making it less straightforward.

LOCODOSY can produce very good results but relies on the segmentation of the mixture spectrum. In the data for sample 2 this was fully automated, but for sample 1 manual intervention was necessary for best results.

It is clear that FDM DOSY can produce similar results to established methods, but with different compromises, and it is expected to be a valuable addition to the current methods available for processing DOSY data.

\section{Acknowledgments}

V.A.M. and B.M. were supported by the National Science Foundation (NSF) Grant No. CHE-1152845. This work was also supported by the Engineering and Physical Sciences Research Council (grant numbers EP/E05899X/1 and EP/ H024336/1). A.A.C. thanks the EPSRC for a DTA studentship.
[1] Johnson, C. S. Jr. Diffusion-ordered nuclear magnetic resonance spectroscopy: principles and applications Prog. Nucl. Magn. Reson. Spectrosc. 34 (1999) 5249-5250

[2] Morris, G.A. Diffusion-Ordered Spectroscopy (DOSY) in Encyclopedia of Magnetic Resonance, eds. R. K. Harris and R. E. Wasylishen, John Wiley and Sons, Ltd, Chichester, UK, 2009. DOI: 10.1002/9780470034590.emrstm0119.pub2.

[3] Barjat, H., G. A. Morris, S. Smart, A. G. Swanson, and S. C. R. Williams. High-Resolution Diffusion-Ordered 2D Spectroscopy (HR-DOSY) - A New Tool for the Analysis of Complex Mixtures J. Magn. Reson. Ser B 108, (1995) 170-93172.
[4] Morris, Kevin F., and Charles S. Johnson. Diffusionordered Two-dimensional Nuclear Magnetic Resonance Spectroscopy J. Am. Chem. Soc. 115 (1992) 3139-3141

[5] Wu, D.H., Chen, A.D., and Johnson, C.S., An Improved Diffusion-Ordered Spectroscopy Experiment Incorporating Bipolar-Gradient Pulses, J. Magn. Reson. Ser. A 115 (1995) 260-264.

[6] Pelta, M.D., Barjat, H., Morris, G.A., Davis, A.L., and Hammond, S.J., Pulse sequences for high-resolution diffusion-ordered spectroscopy (HR-DOSY) Magn. Reson. Chem. 36 (1998) 706-714.

[7] Pelta, M.D., Morris, G.A., Stchedroff, M.J., and Hammond, S.J. A one-shot sequence for high-resolution 
diffusion-ordered spectroscopy Magn. Reson. Chem. 40 (2002) S147-S152.

[8] Nilsson, M., and Morris, G.A., Improving pulse sequences for 3D DOSY: Convection compensation. J. Magn. Reson. 177 (2005) 203-2011.

[9] Botana, A., Aguilar, J.A., Nilsson, M., and Morris, G.A., $J$-modulation effects in DOSY experiments and their suppression: The Oneshot 45 experiment J. Magn. Reson. 208 (2011) 208, 270-278.

[10] Stejskal, E. O.; Tanner, J. E. Spin Diffusion Measurements: Spin Echoes in the Presence of a Time-Dependent Field Gradient J. Chem. Phys. 42 , 288 (1965) 288-292

[11] Connell, M.A., Bowyer, P.J., Adam Bone, P., Davis, A.L., Swanson, A.G., Nilsson, M., and Morris, G.A., Improving the accuracy of pulsed field gradient NMR diffusion experiments: Correction for gradient nonuniformity. J. Magn. Reson. 198 (2009) 121-131.

[12] Barjat, H.; Morris, G. A.; Smart, S.; Swanson, A. G.; Williams, S. C. R.; High-resolution diffusion-ordered $2 D$ spectroscopy (HR-DOSY) - a new tool for the analysis of complex mixtures, J. Magn. Reson. 108 (1995) 170-172.

[13] Nilsson, M.; Connell, M. A.; Davis, A. L.; Morris, G. A. Biexponential fitting of diffusion-ordered NMR data: practicalities and limitations, Anal. Chem. 78 (2006) 3040-3045.

[14] Andrei A. Istratov, A. A.; Vyvenko, O. F. Exponential analysis in physical phenomena Review of Scientific Instruments $\mathbf{7 0}, 2$ (1999) 1233-1257

[15] Provencher, S.W., CONTIN: A general purpose constrained regularization program for inverting noisy linear algebraic and integral equations., Comput. Phys. Commun. 27 (1992) 229-242.

[16] Morris, K. F.; Johnson, C. S.; Resolution of discrete and continuous molecular size distributions by means of diffusion-ordered 2D NMR spectroscopy, J. Am. Chem. Soc. 115 (1993) 4291-4299.

[17] Delsuc, M.A., and Malliavin, T.E. Maximum Entropy Processing of DOSY NMR Spectra, Anal. Chem. 70 (1998) 2146-2148.

[18] Sebastio, R.C.O., Pacheco, C.N., Braga, J.P., and PilVeloso, D. Diffusion coefficient distribution from NMRDOSY experiments using Hopfield neural network J. Magn. Reson. 182 (2006) 22-28.

[19] Day, I. J. On the inversion of diffusion NMR data: Tikhonov regularization and optimal choice of the regularization parameter J. Magn. Reson. 211 (2011) 178185.

[20] Stilbs, P., Paulsen, K., Griffiths, P.C. Global LeastSquares Analysis of Large, Correlated Spectral Data Sets: Application to Component-Resolved FT-PGSE NMR Spectroscopy J. Phys. Chem. 100 , (1996) 81808189

[21] Windig, W.; Antalek, B. Direct exponential curve resolution algorithm (DECRA): A novel application of the generalized rank annihilation method for a single spectral mixture data set with exponentially decaying contribution profiles, Chemom. Intell. Lab. Syst. 37 (1997) 241-254.

[22] Van Gorkom, L. C. M.; Hancewicz, T. M. Analysis of $D O S Y$ and $G P C-N M R$ experiments on polymers by multivariate curve resolution, J. Magn. Reson. 130 (1998) $125-130$

[23] Huo, R.; Wehrens, R.; Buydens, L. M. C. Improved DOSY NMR data processing by data enhancement and combination of multivariate curve resolution with nonlinear least square fitting, J. Magn. Reson. 169 (2004) 257-269.

[24] Nilsson, M.; Morris, G. A.; Speedy component resolution: an improved tool for processing diffusion-ordered spectroscopy data, Anal. Chem. 80 (2008) 3777-3782.

[25] Stilbs, P. RECORD processing - A robust pathway to component-resolved HR-PGSE NMR diffusometry J. Magn. Reson., 207 332-336 (2010)

[26] Colbourne,A. A.; Morris,G.A.; Nilsson, M. Local Covariance Order Diffusion-Ordered Spectroscopy: A Powerful Tool for Mixture Analysis J. Am. Chem. Soc., 133 76407643 (2011)

[27] G. S. Armstrong, N. M. Loening, J. E. Curtis, A. J. Shaka and V. A. Mandelshtam, Processing DOSY spectra using the regularized resolvent transform, J. Magn. Reson. 163 139-148 (2003).

[28] Hansen, P.C. Regularization Tools version 4.0 for Matlab 7.3 Numerical Algorithms, 46 189-194 (207)

[29] Nilsson, M. The DOSY Toolbox: A new tool for processing PFG NMR diffusion data J. Magn. Reson., 200 296-302 (2009)

[30] V. A. Mandelshtam, N.D. Taylor, H. Hu, M. Smith, and A.J. Shaka, Highly resolved double absorption 2D NMR spectra from complex severely truncated $2 \mathrm{D}$ phase modulated signals by filter-diagonalization-averaging method, Chem. Phys. Lett. 305, 209 (1999).

[31] J. Chen, V. A. Mandelshtam and A.J. Shaka, Regularization of the two-dimensional filter diagonalization method: FDM2K, J. Magn. Reson. 146, 363-368, (2000).

[32] V. A. Mandelshtam, FDM: the Filter Diagonalization Method for data processing in NMR experiments, Progress in NMR Spectroscopy, 38, 159-196 (2001).

[33] V. A. Mandelshtam, and H. S. Taylor, Multidimensional harmonic inversion by filter-diagonalization, J. Chem. Phys. 108, 9970 (1998).

[34] P. Thureau, A. Thevand, B. Ancian, G.S Armstrong and V. A. Mandelshtam, Identification of two isomers from an organic mixture by double-stimulated-echo NMR and construction of the DOSY spectra by the regularized resolvent transform method, ChemPhysChem. 6, 15101513 (2005).

[35] M.R. Wall and D. Neuhauser Extraction, through filterdiagonalization, of general quantum eigenvalues or classical normal mode frequenciesfromasmallnumberofresidues or a shorttime segment of a signal. I. Theory and application to a quantumdynamics model $J$. Chem. Phys. 102, 8011 (1995). 